\title{
Firing Properties of Anatomically Identified Neurons in the Medial Septum of Anesthetized and Unanesthetized Restrained Rats
}

\author{
Axelle Pascale Simon, Frédérique Poindessous-Jazat, Patrick Dutar, Jacques Epelbaum, and Marie-Hélène Bassant \\ Institut National de la Santé et de la Recherche Médicale (INSERM) Unité Mixte de Recherche 549, Faculté de Médecine, Université Paris René- \\ Descartes, 75014 Paris, France
}

Cholinergic and GABAergic neurons in the medial septum-diagonal band of Broca (MS-DB) project to the hippocampus where they are involved in generating theta rhythmicity. So far, the functional properties of neurochemically identified MS-DB neurons are not fully characterized. In this study, MS-DB neurons recorded in urethane anesthetized rats and in unanesthetized restrained rats were labeled with neurobiotin and processed for immunohistochemistry against glutamic acid decarboxylase (GAD), parvalbumin (PV), and choline acetyltransferase (ChAT). The majority of the 90 labeled neurons (75.5\%) were GAD + . Among them, $34.0 \%$ were also PV + , but none were ChAT + . Only $8.8 \%$ of the labeled neurons were found ChAT + . Remaining neurons (15.5\%) were not identified. In anesthetized rats, all of the PV/GAD + and 65\% of GAD + neurons exhibited burst-firing activity at the theta frequency. PV/GAD + neurons displayed higher discharge rate and longer burst duration compared with GAD + neurons. At variance, all of the ChAT + neurons were slow-firing. Cluster-firing and tonic-firing were observed in GAD + and unidentified neurons. In unanesthetized rats, during wakefulness or rapid eye movement sleep with hippocampal theta, the bursting neurons were PV/GAD + or GAD +, whereas all of the ChAT+ neurons were slow-firing. Across the sleep-wake cycle, the GABAergic component of the septohippocampal pathway was always more active than the cholinergic one. The fact that cholinergic MS-DB neurons do not display theta-related bursting or tonic activity but have a very low firing rate questions how acetylcholine exerts its activating role in the septohippocampal system.

Key words: septohippocampal; theta rhythm; burst-firing; cholinergic; GABA; parvalbumin

\section{Introduction}

The medial septum-diagonal band of Broca complex (MS-DB) plays a key role in generating the hippocampal theta, an oscillatory activity implicated in learning and memory (Buzsaki, 2002). The septohippocampal pathway is composed of cholinergic, GABAergic, and glutamatergic neurons (Köhler et al., 1984; Frotscher and Leranth, 1985; Manns et al., 2001). The large majority of GABAergic septohippocampal neurons contain the calciumbinding protein parvalbumin (PV) (Freund, 1989). A significant number of MS-DB neurons exhibit a rhythmically bursting (RB) activity that is considered the major generator of hippocampal theta (Dutar et al., 1986; Stewart and Fox, 1989; King et al., 1998; Brazhnik and Fox, 1999; Borhegyi et al., 2004). Numerous in vivo studies attempted to establish the nature of the RB septal neurons, but, until recently, the correlation between neurochemical phenotypes and firing properties remained indirect. Using juxtacellular recording and labeling with neurobiotin, it was shown that PV immunoreactive $(\mathrm{PV}+)$ neurons exhibited bursting activity tightly coupled to hippocampal theta waves (Borhegyi et al., 2004; Bassant et al., 2005). $\mathrm{RB}$ activity was also observed in GABAergic neurons found to ex-

Received April 3, 2006; revised July 5, 2006; accepted July 20, 2006.

Correspondence should be addressed to Marie-Hélène Bassant, Institut National de la Santé et de la Recherche Médicale Unité 549, Institut Fédératif de Recherche IFR 77 Broca Ste Anne, 2 ter rue d'Alésia, 75014 Paris, France. E-mail: bassant@broca.inserm.fr.

DOI:10.1523/JNEUROSCI.1401-06.2006

Copyright $\odot 2006$ Society for Neuroscience $\quad$ 0270-6474/06/269038-09\$15.00/0 press glutamic acid decarboxylase (GAD) but not PV (Bassant et al., 2005). These data demonstrated undoubtedly that the GABAergic MS-DB neurons are capable of displaying bursting activity at theta frequency.

In contrast, the firing properties of cholinergic MS-DB neurons remained to be characterized in vivo. In MS-DB slice preparations, cholinergic neurons identified by acetylcholinesterase (AChE) histochemistry or choline acetyltransferase (ChAT) immunohistochemistry (Griffith and Matthews, 1986; Markram and Segal, 1990) and reverse transcription-PCR (Sotty et al., 2003) display electrophysiological properties such as longduration afterhyperpolarization (AHP) and slow firing rates that limit their capacity for RB activity. Such characteristics suggest that the burst-firing neurons recorded in MS-DB cannot be cholinergic. This, however, appeared at odds with in vivo data. For example, long-duration AHP-cells displaying a theta-related RB abolished by muscarinic antagonists were assumed to be cholinergic (Brazhnik and Fox, 1997). Also, the loss of bursting activity in MS-DB after the selective lesion of cholinergic septohippocampal neurons was interpreted as a proof that these cells were rhythmic cells (Apartis et al., 1998). At the present time, a widely accepted concept is that the bursting MS-DB neurons comprise the GABAergic ones, which pace the theta activity, and the cholinergic ones, which contribute to its amplitude (Stewart and Fox, 1990; Lee et al., 1994; Apartis et al., 1998; Brazhnik and Fox, 1999). This concept, however, remains speculative, and in vivo 
data are required to clarify the role of cholinergic neurons in the septohippocampal network and more specifically in the hippocampal theta generation.

In the present experiment, MS-DB neurons recorded in anesthetized and unanesthetized rats were labeled with neurobiotin and subsequently processed for immunohistochemistry for GAD, PV, and ChAT. The study aimed to: (1) characterize the discharge profiles of GAD and PV immunoreactive neurons, (2) examine whether their activity change across the sleep/wake cycle, and (3) to unravel the firing patterns of identified cholinergic neurons.

\section{Materials and Methods}

Thirty-seven male Sprague Dawley rats (270-300 g body weight; IffaCredo, L'Arbresle, France) were used in the urethane-anesthetized state and 18 in unanesthetized state. Rats were housed in our animal facility in conformity with the local Animal Care and Use Committee. Experiments were performed according to the guidelines of the French Act of Animal Care and Experimentation (1990; registration number 75-343). All efforts were made to minimize pain and suffering and to reduce the number of animals used.

\section{Anesthetized rats}

Rats were anesthetized with urethane $(1.3 \mathrm{~g} / \mathrm{kg}$, i.p. $)$ and placed in a stereotaxic frame. A catheter was inserted in the peritoneal cavity to give small additional doses of urethane if necessary to keep the rat at a level of anesthesia optimal for recording the hippocampal theta rhythm. In addition to the general anesthesia, all wounds and pressure points were infiltrated with lidocaine before surgery. Body temperature was maintained with a heating pad controlled by a rectal thermal probe. To record electroencephalogram (EEG), a monopolar electrode was placed in the left hippocampus [anteroposterior (AP), 5; lateral (L), 3; height (H), 3.2; stratum lacunosum-moleculare) (Paxinos and Watson, 1986). A screw placed on the occipital crest served as reference. The skull was opened above the MS-DB (AP, 9.5; L, 0), and the central sinus was ligatured and cut.

\section{Unanesthetized rats}

The head-restraining system consists of a U-shaped piece of aluminum designed to be cemented to the rat skull. Four bolts in each angle of the $U$ piece allow fixing it to a sliding carriage adapter, itself fastened to the stereotaxic apparatus. This device allows a painless restraint of the rat's head with high mechanical stability (Souliere et al., 2000). Rats were anesthetized with pentobarbital $(60 \mathrm{mg} / \mathrm{kg})$ and placed in a stereotaxic frame. The surgery was similar to the one described above for the urethane anesthetized rats, except that a stainless steel hook soldered to a fine flexible insulated silver wire was placed in the neck muscles to record electromyogram (EMG). A liquid bonding resin (Superbond; Sun Medical, Kyoto, Japan) was applied on the cleaned, dried skull surface. The carriage adapter was set so that the U-shaped piece was centered over the MS-DB region. The electrodes were soldered to a connector. The U-shaped piece and the electrodes were embedded in dental cement and firmly jointed to the rat skull. The opening over the MS-DB was covered by gelfoam soaked in saline. The well formed by the cement ridges was covered with sterile bone wax. The rat was removed from the stereotaxic frame and allowed to recover from anesthesia. Intramuscular cephalosporin (cefuroxime, $60 \mathrm{mg} / \mathrm{kg}$; GlaxoSmithKline, Brentfor, UK) was administered every $2 \mathrm{~d}$ during the recovery period.

\section{Habituation to head fixation}

Rats were habituated to the restraint system during 7-10 d. The rat's head was painlessly secured to the stereotaxic frame while its body was comfortably supported by a hammock. The length of the training sessions increased gradually (from 5-10 min to $2 \mathrm{~h}$ ). A piece of cucumber or a cookie was given to the rat after each session. Over a course of $\sim 3 \mathrm{~d}$, the rat stood motionless and fell asleep easily. At the end of the training period, the rats slept the majority of the time, and rapid eye movement (REM) sleep can be observed attesting that the immobilization was well tolerated. The head restraining system let the rat feed, drink, and sleep when returned to its cage after the training session (Apartis et al., 1998).

\section{Electrophysiology}

Single-unit and polygraphic recordings. In both urethane anesthetized and unanesthetized rats, single units were recorded in the midline part of MS-DB (L 0 to L 0.4 , left and right sides) using glass microelectrodes (10-15 M $\Omega$ ) filled with $0.5 \mathrm{M}$ sodium acetate and 5\% neurobiotin (Vector Laboratories, Burlingame, CA). When two cells were filled with neurobiotin in the same rat, they were located in clearly distinct areas (left or right side, dorsal or ventral MS-DB) to ensure unambiguous identification of the cells. Neuronal signal was amplified by a DC amplifier (Axoclamp 2B; Molecular Devices, Foster City, CA), filtered between 0.1 and 5 $\mathrm{kHz}$ by a signal conditioner (Cyberamp 380; Molecular Devices) and digitized at $10 \mathrm{kHz}$ (Cambridge Electronic Design, Cambridge, UK). Recordings with a high signal-to-noise ratio $(>4)$ were selected so that neuron singularity could be easily assessed by a window discriminator. Once a single neuron was isolated, discharge rate, spike duration, and spontaneous or induced RB activity were examined on-line and then recorded for $>5 \mathrm{~min}$. The electrode was then progressively advanced to reach close proximity of the cell. Pulses of anodal current (200 ms; 50\% duty cycle) were delivered by way of microelectrode. The current amplitude was increased incrementally from $0.4 \mathrm{nA}$ to a maximum of $10 \mathrm{nA}$ until the cell firing became entrained for 3-10 min (Pinault, 1996).

In unanesthetized rats, each experiment lasted for a maximum of three consecutive days, depending on the opportunity to record and to label a cell of interest. A maximum of three recording sessions of 2-3 h were performed each day from 11:00 A.M. to 6:00 P.M. The state of arousal was continuously monitored (EEG and EMG) while single-unit activity was recorded. Four states were differentiated: wakefulness (W) (highfrequency, low-voltage EEG activity intermingled with periods of hippocampal theta, if necessary induced by auditory stimulation or slight pressure on tail, sustained EMG activity), slow-wave sleep (SWS) (highamplitude slow waves intermingled with spindles), and REM sleep (marked theta activity $6-8 \mathrm{~Hz}$, loss of neck muscles tones, jerks of jaw muscles, and vibrissae at the end of the episode). Cells were recorded during periods of time sufficient to observe at least two different states of arousal and then labeled with neurobiotin.

Data analysis. Signals were collected on a personal computer via a Cambridge Electronic Design interface using the Spike 2 software (sampling rate, $1 \mathrm{kHz}$ ) in parallel with analog-to-digital samplings of filtered EEG (bandpass, 1-70 Hz; sampling rate, $100 \mathrm{~Hz}$ ). The power spectrum of EEG (10 s epochs) was computed with fast Fourier transform over a range of $0-25 \mathrm{~Hz}$ with $0.1 \mathrm{~Hz}$ of resolution. The unit activity was analyzed per $10 \mathrm{~s}$ epoch for: (1) average discharge rate (number of spike/s); (2) duration of spike (measured from the peak of initial negative phase of the action potential to the peak of its subsequent positive phase) (Brazhnik and Fox, 1999); (3) instantaneous firing frequency $(f)_{1}$ [i.e., the first modal peak of the histogram of interspike interval (ISIH; 55 bins of $5 \mathrm{~ms}$ )]; and (4) autocorrelation histogram (HAC) use to characterize the RB pattern of activity. If recurrent events such as rhythmic burst of action potentials occur, then the autocorrelation histogram shows periodic sinusoidal-like density peaks (Perkel et al., 1967). An "index" of rhythmic activity (average value of peaks minus average value of troughs divided by average discharge rate) was used to classify cells as RB or non-RB neurons (Apartis et al., 1998). Cells with an index below 0.95 were classified as nonrhythmic. The higher index denotes the more regular RB activity. In addition, the duration of the burst and the number of spikes per burst were calculated.

Based on the parameters mentioned above, four patterns of discharge were distinguished: burst-firing (2-20 spike/burst; intraburst interval, $\leq 30 \mathrm{~ms}$; interburst interval, $\geq 60 \mathrm{~ms}$; bimodal ISIH, rhythmicity index, $\geq 0.95$ ); cluster-firing [irregular clusters of few spikes; average discharge rate, $\leq 5$ spike/s; abscise of $(f)_{1} \mathrm{ISIH} \leq 25 \mathrm{~ms}$ ); tonic-firing [average discharge rate $>10$ spike/s, ISIH monomodal; abscise of $(f)_{1} \mathrm{ISIH} \geq 25 \mathrm{~ms}$ ] flat HAC, slow-firing (average discharge rate $<4$ spike/s). Neurons that changed their discharge from tonic to burst-firing pattern in response to theta induction were classified as RB neurons. As previously reported (Sweeney et al., 1992), fast-firing tonic neurons can be provoked to firing rhythmically but not neurons with low discharge rates (cluster and slow-firing neurons). 


\section{Neuronatomy}

Primary antibodies. Cholinergic cells were detected with a goat antiserum directed against ChAT (AB144P; 1:100; Chemicon, Temecula, CA). GABAergic cells were detected with a polyclonal rabbit antibody, which preferentially recognizes the larger form $(67 \mathrm{KDa})$ of GAD67 but also GAD65 (AB5992; 1/500; Chemicon). A monoclonal antibody directed against the calcium binding protein PV (P3088; 1:500; Sigma, St Louis, MO) was also used.

\section{Tissue preparation}

Rats were killed 1-6 h after the labeling of neurons. The unanesthetized rats were deeply anesthetized with sodium pentobarbital $(60 \mathrm{mg} / \mathrm{kg}$ i.p.; Sanofi Pharmaceuticals, Toulouse, France). All of the animals were perfused through the ascending aorta with $600 \mathrm{ml}$ of $4 \%$ paraformaldehyde, $2 \%$ picric acid in $0.1 \mathrm{M}$ phosphate buffer $(\mathrm{PB}), \mathrm{pH}$ 7.4. Brains were cryoprotected in $30 \%$ sucrose in $\mathrm{PB}\left(4^{\circ} \mathrm{C}, 24 \mathrm{~h}\right)$, frozen in liquid isopentane at $-35^{\circ} \mathrm{C}$, coronally sectioned at a thickness of $40 \mu \mathrm{m}$ at the level of the MS-DB (AP, 9.9 to -8.8) (Paxinos and Watson, 1986), and collected in PB.

\section{Neurobiotin detection}

For the detection of neurobiotin-injected neurons, the septal freefloating sections were incubated in Streptavidin CY3 (1:16000; Jackson ImmunoResearch, West Grove, PA) in Tris buffered saline (TBS), pH 7.4 , for $30 \mathrm{~min}$. They were then rinsed in TBS, mounted on glass slides, and coverslipped with Vectashield (Vector Laboratories). The red fluorescent-labeled cells were located with a Zeiss (Thornwood, NY) Axioplan 2 microscope under epifluorescence illumination.

\section{Immunohistochemistry}

To characterize the neurochemical identity of neurobiotin-containing neurons, the relevant sections were demounted, rinsed in TBS, and blocked by preincubation in 5\% normal donkey serum in TBS for 30 min. When cells were expected to be cholinergic (based on electrophysiological parameters), sections were incubated in a mixture of goat antiChAT and rabbit anti-GAD primary antibodies for $48-72 \mathrm{~h}$ at room temperature and revealed with a mixture of Alexa 488-conjugated donkey anti-goat (1:100; Invitrogen, Eugene, OR), and CY5-conjugated donkey anti-rabbit IgG (1/100; Jackson ImmunoResearch) for $2 \mathrm{~h}$; when cells were expected to contain $\mathrm{PV}$, sections were incubated in a mixture of mouse anti-PV and rabbit anti-GAD and detected with Alexa 488conjugated donkey anti-mouse $\operatorname{IgG}$ (1:100; Invitrogen), mixed with CY5-conjugated donkey anti-rabbit IgG. They were then rinsed, mounted, and coverslipped. To ensure detection of all phenotypes, when neurobiotin-labeled cells were negative for green-revealed ChAT, sections were reincubated with mouse anti-PV and newly green-revealed with Alexa 488-conjugated anti-mouse; when cells were negative for PV, sections were reincubated with goat anti-ChAT and newly greenrevealed with Alexa 488-conjugated anti-goat. Control incubations performed on sections containing ectopically labeled cells included omission of the primary antibodies to test the nonspecific binding of secondary antibodies and incubation with one primary but with secondary antibodies to demonstrate the absence of cross-labeling in doubleand triple-labeling experiments.

The CY3/A488 double-labeled cells (neurobiotin/ChAT+ or PV+) were detected by epifluorescence illumination and photographed with a Photometrics (Huntington Beach, CA) camera.

The CY5-labeled cells (GAD+) were detected by confocal laserscanning microscopy using a TCS SP 2 confocal imaging system equipped with HeNe $543 \mathrm{~nm}$ and HeNe $633 \mathrm{~nm}$ lasers (Leica Microsystems, Heidelberg, Germany). Digital images were collected from a single optical plane using a $40 \times$ Plan-Apochromat oil immersion lens (numerical aperture, 1.25). Pinhole setting was 1 Airy unit (AU) for all images. Double fluorescence images were acquired in sequential mode to avoid potential contamination by linkage-specific fluorescence emission cross talk.

\section{Results}

Immunohistochemical identification of juxtacellular-labeled neurons

Ninety neurons were successfully recorded and labeled (62 in anesthetized rats and 28 in unanesthetized rats). The labeled neu-

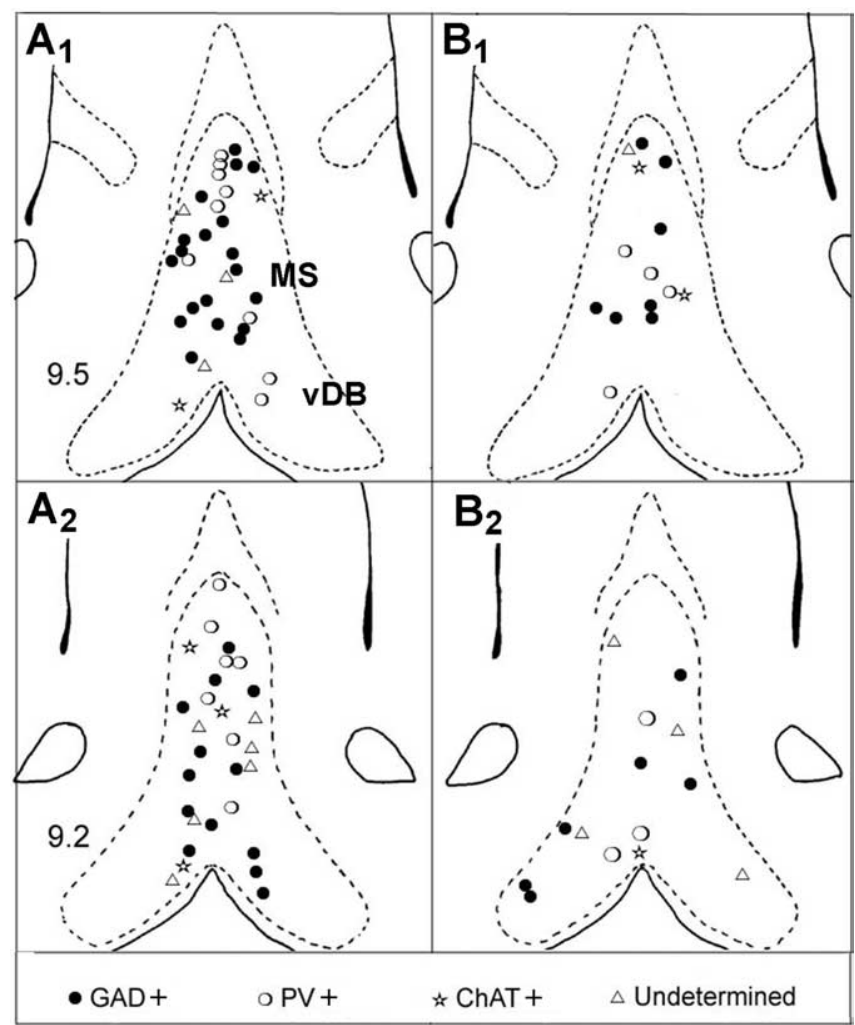

Figure 1. Location of neurobiotin-labeled neuron in the medial septum (MS) and the vertical limb of the diagonal band of Broca (VDB) in anesthetized $(\boldsymbol{A 1}, \boldsymbol{A 2})$ and unanesthetized $(\boldsymbol{B} 1, \boldsymbol{B} 2)$ rats. Each symbol represents the location of one neuron in two coronal sections of MS-DB (interaural 9.5 and 9.2) [adapted from the atlas of Paxinos and Watson (1986)].

rons were numerous in the MS and fewer in the vertical limb of the DB (Fig. 1). Their profiles ranged from small size $(10-15 \mu \mathrm{m})$ with ovoid or fusiform shape to medium (15-20 $\mu \mathrm{m})$ or large size (up to $25 \mu \mathrm{m}$ ) with polygonal shape. Ovoid and polygonal cells were more numerous than fusiform cells (41.1, 38.9, and 20\%, respectively), but the repartition of morphological types did not differ significantly between the groups defined from neurochemical criteria. Of the 90 labeled neurons, 68 (75.5\%) expressed GAD immunoreactivity. Among these 68 neurons, 43 (66\%) expressed only GAD (GAD+) and $23(34 \%)$ expressed also PV immunoreactivity $(\mathrm{PV} / \mathrm{GAD}+)$, but none was ChAT + . Only eight neurons of $90(8.8 \%)$ expressed ChAT immunoreactivity. Fourteen neurons (15.5\%) did not express immunoreactivity for either GAD, PV, or ChAT. They were classified as undetermined neurons. Although the total number of neurobiotin-labeled neurons was different in the two experimental groups, the percentages of GABAergic, cholinergic, and undetermined neurons were found similar in anesthetized rats and unanesthetized rats.

\section{Electrophysiological properties of identified neurons} Anesthetized rats

Labeled cells were classified according to their neurochemical identity, their firing patterns (see Materials and Methods), and the presence or absence of hippocampal theta rhythm (Table 1). The repartition of neurons in terms of shapes did not differ significantly between groups defined on electrophysiological criteria

GAD+ neurons

Of $23 \mathrm{GAD}+$ neurons recorded during hippocampal theta rhythm, 15 (65.2\%) discharged in burst of spikes (Fig. 2). The mean discharge rate of the $\mathrm{GAD}+$ burst-firing neurons was 
Table 1. Firing patterns of neurons of various neurochemical phenotypes

\begin{tabular}{|c|c|c|c|c|c|}
\hline & & $\mathrm{GAD+}$ & $\mathrm{PV} / \mathrm{GAD}+$ & ChAT+ & Undetermined \\
\hline \multirow[t]{7}{*}{$\begin{array}{l}\text { Neurons recorded during } \\
\text { hippocampal theta }(n=46)\end{array}$} & Burst-firing & $\begin{array}{l}18.3 \pm 2.0 \\
15 / 23\end{array}$ & $\begin{array}{l}26.5 \pm 2.5^{*} \\
12 / 12\end{array}$ & & \\
\hline & Tonic-firing & $27.1 \pm 0.6$ & & & $15.6 \pm 3.2$ \\
\hline & & $2 / 23$ & & & $2 / 7$ \\
\hline & Cluster-firing & $8.6 \pm 1.3$ & & & $12.9 \pm 6.1$ \\
\hline & & $4 / 23$ & & & $2 / 7$ \\
\hline & Slow-firing & $3.0 \pm 0.5$ & & $0.6 \pm 0.3$ & $1.4 \pm 0.9$ \\
\hline & & $2 / 23$ & & $4 / 4$ & $3 / 7$ \\
\hline \multirow{7}{*}{$\begin{array}{l}\text { Neurons recorded in the absence of } \\
\text { hippocampal theta }(n=16)\end{array}$} & Burst-firing & & & & \\
\hline & Tonic-firing & $16.5 \pm 3.2$ & $23.0 \pm 5.0$ & & \\
\hline & & $3 / 9$ & $2 / 4$ & & \\
\hline & Cluster-firing & $11.1 \pm 2.5$ & & & 7.0 \\
\hline & & $6 / 9$ & & & $1 / 2$ \\
\hline & Slow-firing & & $2.3 \pm 0.3$ & 0.3 & 1.0 \\
\hline & & & $2 / 4$ & $1 / 1$ & $1 / 2$ \\
\hline
\end{tabular}

Data are from urethane-anesthetized rats. Bolded values indicate the number of neurons. Other values are average discharge rates (spikes/s) \pm SEM. ${ }^{*} p<0.05$ compared with GAD-IR burst-firing neurons.

$18.3 \pm 2.0 \mathrm{spike} / \mathrm{s}$. The average frequency of the bursts was $4.0 \pm$ $0.08 \mathrm{~Hz}$. Bursts were composed of $4.8 \pm 0.50$ brief spikes (duration, $0.49 \pm 0.03 \mathrm{~ms}$ ) and had a mean length of $68.7 \pm 2.8 \mathrm{~ms}$. The rhythmicity index, calculated from HAC, was $1.7 \pm 0.15$. ISIHs were bimodal with a first modal peak of $41.8 \pm 11$ spike/bin.

Other discharge profiles less often encountered were clusterfiring $(n=4)$, tonic-firing $(n=2)$, and slow-firing $(n=2)$ (Table $1)$. Tonic-firing neurons had a higher discharge rate $(27.0 \pm 6.0$ spike/s) and a shorter spike duration ( $0.38 \pm 0.02 \mathrm{~ms})$ compared with cluster-firing $(8.6 \pm 1.3$ spike/s and $0.59 \pm 0.05 \mathrm{~ms})$ and slow-firing ( $3.0 \pm 0.5 \mathrm{spike} / \mathrm{s}$ and $0.75 \pm 0.15 \mathrm{~ms})$ neurons.

None of the GAD + neurons recorded in the absence of hippocampal theta were burst-firing. Three were tonic (16.5 \pm 3.2 spike/s), and six were cluster-firing ( $11.1 \pm 2.5$ spike/s $)$ neurons. The large majority of GAD + neurons $(81 \%)$ had short-duration spike, but some of them (one burst-firing and five cluster-firing) had long duration spike ( $\geq 0.7 \mathrm{~ms}$ ).

\section{$P V / G A D+$ neurons}

All of the PV/GAD + neurons recorded during hippocampal theta rhythm $(n=12)$ exhibited burst-firing pattern of activity (frequency, $4.1 \pm 0.13 \mathrm{~Hz}$ ) (Table 1). Compared with the GAD+ bursting neurons, $\mathrm{PV} / \mathrm{GAD}+$ neurons had a higher discharge rate $(26.5 \pm 2.5$ spike/s; $p<0.05)$, a longer burst duration (107.4 $\pm 8.4 \mathrm{~ms} ; p<0.001)$, a higher number of spikes per burst $(8.0 \pm 0.7 ; p<0.001)$, and a higher first modal peak of ISIH $(85.4 \pm 13$ spike/bin; $p<0.05)$ (Fig. 2). The bursting activity was strikingly regular, as shown by the high values of the rhythmicity index ( $3.0 \pm 0.4 ; p<0.01$; compared with GAD + neurons). An overlap of the discharges profiles between $\mathrm{PV} / \mathrm{GAD}+$ and GAD + neurons was observed in three cases, indicating that electrophysiological properties alone were not sufficient to predict the chemical identity of a cell.

None of the PV/GAD + neurons recorded in the absence of hippocampal theta were burst-firing (Table 1). Two neurons were tonic, with a discharge rate $(23.0 \pm 5.0$ spike/s, n.s., compared with PV/GAD + burst-firing neurons), and two were slowfiring $(2.3 \pm 0.3 \mathrm{spike} / \mathrm{s})$. All of the PV/GAD + neurons had brief spikes $(0.42 \pm 0.03 \mathrm{~ms})$.

\section{ChAT+ neurons}

Intracellular recordings in the septal slice preparation showed that ChAT + neurons were slow firing with a broad action poten- tial and long-duration AHP (Markram and Segal, 1990). It was further suggested that the cholinergic neurons could also be identified using extracellular recording methods on the basis of their slow firing, broad action potentials and "hump" in the falling phase of the action potential (Matthews and Lee, 1991). Although an unquestionable identification of neurons cannot be inferred from extracellular signal, an attempt was made to record and label slow firing cells (spontaneous activity, $<4$ spike/s) with spike duration $>0.8$ $\mathrm{ms}$. Of nine slow-firing cells recorded in the presence of hippocampal theta, four were $\mathrm{ChAT}+$, two were $\mathrm{GAD}+$, and three were undetermined. One slow-firing cell recorded and labeled in the absence of hippocampal theta was also found ChAT+. The five ChAT + neurons had a very low level of discharge $(0.5 \pm 0.3$ spike/s $)$ and a long duration spike $(0.84 \pm 0.04 \mathrm{~ms})$ with a hump on the falling phase of the spike (Fig. 3). Taking into consideration that none of the labeled neurons with rhythmically bursting, tonic, and or cluster activity expressed ChAT + and that ChAT immunoreactivity was found only in slow firing cells, our results strongly suggest that cholinergic MS-DB neurons are characterized by a very low level of discharge, even when theta activity is present in the hippocampus.

\section{Undetermined neurons}

None of the undetermined neurons $(n=9)$ recorded during or in the absence of hippocampal theta were burst-firing units (Table 1). Two were tonic-firing (spike duration, $0.40 \mathrm{~ms}$ ), three were cluster-firing (spike duration, $0.53 \pm 0.13 \mathrm{~ms}$ ), and four were slow-firing cells with long-duration spike $(0.71 \pm 0.06 \mathrm{~ms})$.

\section{Unanesthetized rats}

Of the 28 labeled neurons, 10 were recorded across W, SWS, and REMs, 18 during W/SWS or SWS/REMs. Neurons were classified according to their patterns of discharge and the vigilance state (Table 2). Neurons that displayed rhythmic bursting during W and/or REMs were classified as burst-firing neurons, although they did not discharge rhythmically during SWS.

\section{GAD + neurons}

Thirteen labeled neurons expressed GAD immunoreactivity (Table 2). All had brief spike duration $(0.40 \pm 0.03 \mathrm{~ms})$. Three neurons exhibited rhythmic bursting during $\mathrm{W}$ and REMs $(5.7 \pm$ $0.22 \mathrm{~Hz}$ during $\mathrm{W} ; 6.9 \pm 0.49 \mathrm{~Hz}$ during REMs). Burst parameters did not differ significantly between W (burst length, $63.2 \pm$ $1.0 \mathrm{~ms}$; spike/burst, $6.2 \pm 1.0$ ) and REMs (burst length, $65.4 \pm$ $11.0 \mathrm{~ms}$; spike/burst, $8.2 \pm 2.1$ ). The rhythmically bursting activity, however, was more sustained during REMs compared with W as shown by the higher value of RI (2.13 vs 1.13 , respectively). Bursting activity was no longer observed during SWS. Six neurons were cluster-firing and retained this pattern across sleepwake cycle (Fig. 4). The average discharge rate decreased during SWS, but the difference did not reach a significant level (paired $t$ test; $p=0.10$ ). Four GAD + neurons were tonic-firing with a similar discharge rate during $\mathrm{W}$ and SWS. One tonic-firing neuron recorded during the three states exhibited a higher discharge rate during REMs. 
PV/GAD + neurons

Seven labeled neurons expressed PV immunoreactivity (Table 2). Six neurons exhibited burst-firing pattern of activity $(5.7 \pm 0.10 \mathrm{~Hz}$ during $\mathrm{W} ; 6.3 \pm 0.03 \mathrm{~Hz}$ during REMs; $p<0.01)$. Burst parameters did not differ significantly between $\mathrm{W}$ (burst length, $75.0 \pm 4.7 \mathrm{~ms}$; spike/ burst, $6.7 \pm 0.5$ ) and REMs (burst length, $75.8 \pm 2.5 \mathrm{~ms}$; spike/burst, $7.4 \pm 1.0)$. As observed for GAD + neurons, the rhythmically bursting activity was more sustained during REMs compared with W (RI 2.80 vs 1.90 ) and no longer observed during SWS (Fig. 5). One neuron was cluster-firing during W and SWS.

\section{ChAT+ neurons}

The three ChAT + neurons recorded during W and SWS were slow-firing (Table 2). As observed previously in anesthetized rats, they displayed a very low discharge rate (Fig. 2), which contrasted with the high discharge rates of GAD + and PV/ $\mathrm{GAD}+$ neurons. They had a long duration spike $(0.70 \pm 0.05 \mathrm{~ms})$ with a hump on the falling phase of the spike.

\section{Undetermined neurons}

Of the five undetermined neurons recorded during $\mathrm{W}$ and SWS, two were tonic-firing with brief spike and high discharge rates, and three were slowfiring (spike duration, $0.67 \pm 0.13 \mathrm{~ms}$ ) (Table 2).

\section{Comparison between the} experimental conditions

The average discharge rate of neurons recorded in unanesthetized rats $(n=28)$ was higher $(24.0 \pm 2.3$ spike/s $)$ than those of neurons recorded in urethane condition $(n=62)(15.0 \pm 1.4$ spike $/ \mathrm{s}$; $p<0.01)$. It was worth noting that the firing rate increased from W $(19.7 \pm 2.7$ spike/s) to SWS (23.0 \pm 3.5 spike/s $)$ and to REMs (36.6 \pm 6.7 spike/s). Grouping neurons according to their firing pattern and chemical identity resulted in fewer neurons per group (Tables 1, 2). Thus, differences reached a significant level only in two cases: in burst-firing PV/ $\mathrm{GAD}+$ neurons during REMs $(p<$ $0.05)$ and in cluster-firing GAD + neurons during SWS $(p<0.05)$.

In terms of discharge profiles of identified neurons, results obtained in unanesthetized rats agreed with those obtained in urethane condition. They showed that only GAD + and PV/ $\mathrm{GAD}+$ neurons displayed bursting activity and that cluster firing was most often observed in GAD + neurons. Interestingly, the unanesthetized approach allowed confirming that ChAT + neurons had a very low discharge rate even during wakefulness with hippocampal theta.
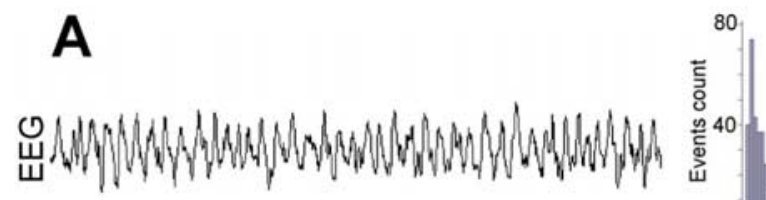

HAC $\mathrm{RI}=2.5$

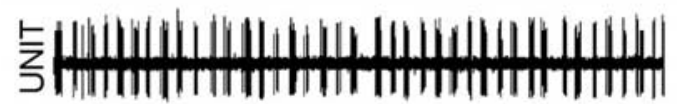

B

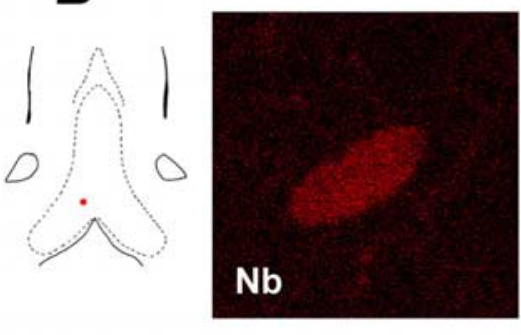

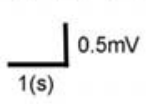

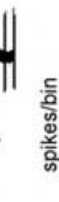

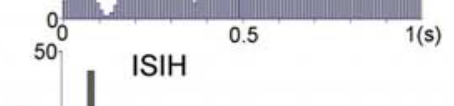

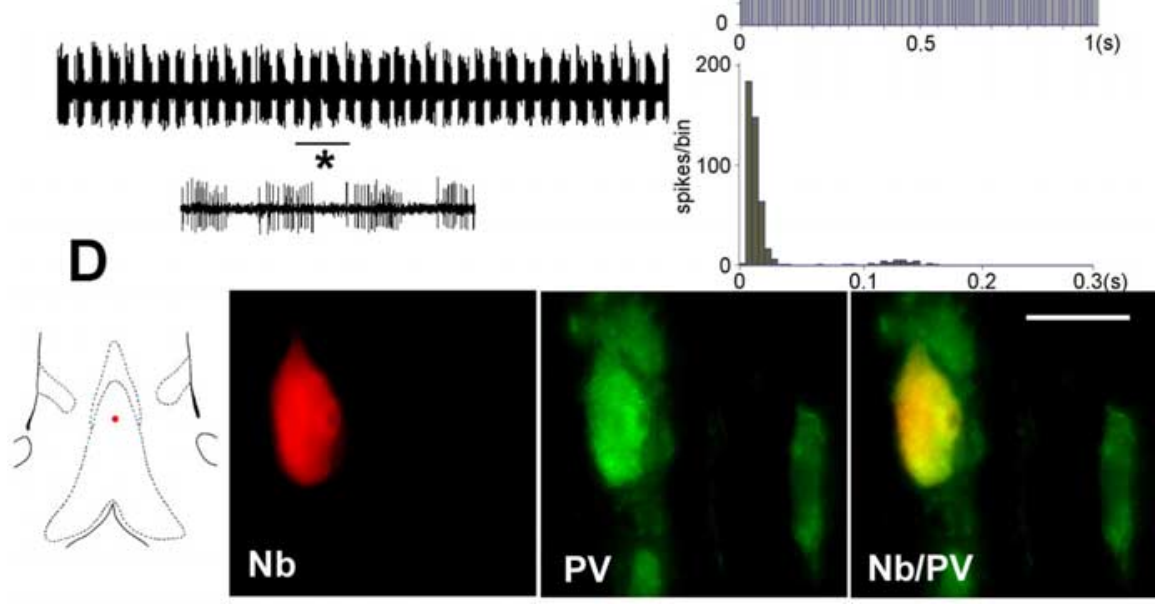

Figure 2. Discharge properties of two GABAergic bursting neurons recorded in MS-DB of urethane anesthetized rats in the presence of hippocampal theta (EEG). $A, B, G A D+$ neuron. $C, D, P V / G A D+$ neuron. The red dots on the frontal sections of MS-DB show the location of the neurons. The rhythmically bursting pattern of the neurons is clearly shown by the aspect of the unit activity (10 s epochs), the periodic peaks of the HAC and the bimodal profile of the histogram of ISIHs. Note that the PV/GAD + neuron (C) displays a higher discharge rate compared with the GAD + neuron $(\boldsymbol{A})$. Its rhythmically bursting activity is more pronounced as shown by the higher values of the RI. As evident in 1 s expended traces (asterisk), the number of spikes per burst is higher in the PV/GAD + neuron compared with the GAD + neuron (11-18 and 3-5, respectively). $\boldsymbol{B}$, Single confocal sections showing the neuron in $\boldsymbol{A}$ labeled with neurobiotin (Nb) (Cy3, red). This neuron was also immunoreactive for GAD (Cy5, false blue color). $\boldsymbol{D}$, Microphotograph showing the neuron in $\boldsymbol{C}$ labeled with neurobiotin (red). This neuron was also immunoreactive for PV (Alexa 488; green). Scale bars, $20 \mu \mathrm{m}$. 


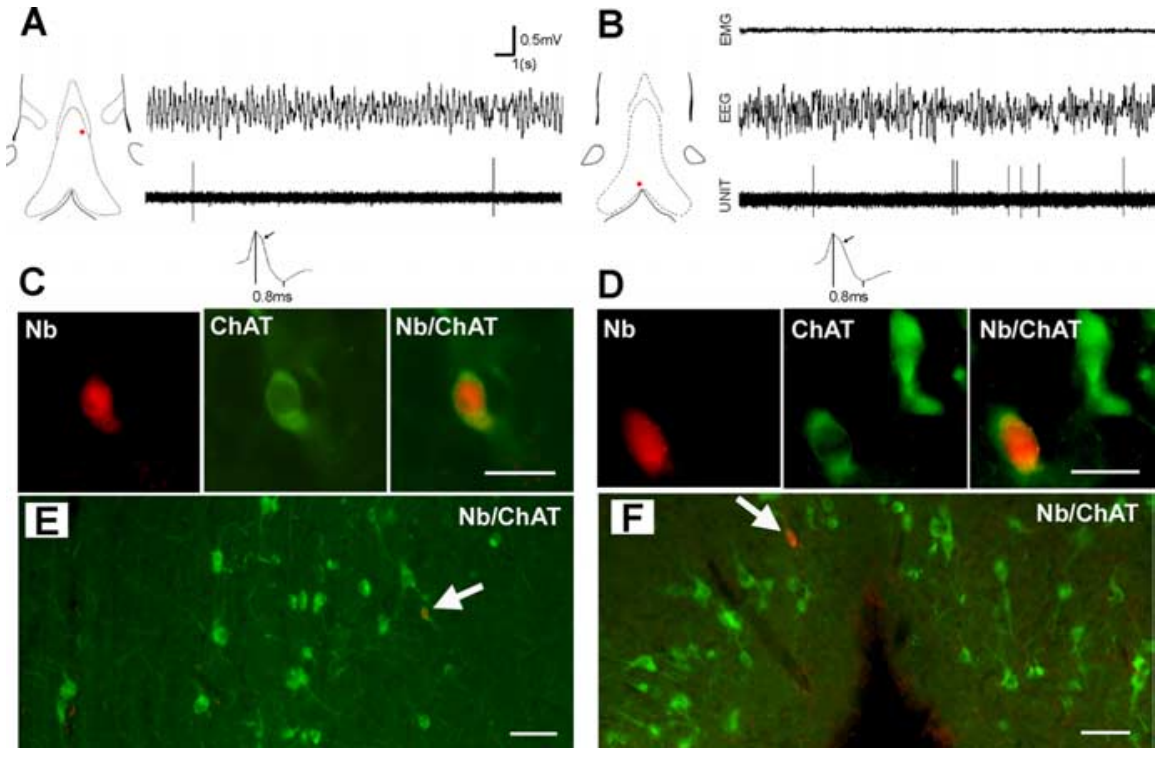

Figure 3. Discharge properties of two cholinergic neurons recorded in an anesthetized rat $(\boldsymbol{A})$ and in an unanesthetized rat $(\boldsymbol{B})$. The red dots on the frontal sections of MS-DB show the location of the neurons. Note the low level of discharge of both units ( 20 s epochs) in the presence of urethane-induced or wake-related hippocampal theta (EEG). On expended traces, note the hump on the falling phase of the potential (arrow) and long duration of the spike (0.8 ms). C, D, Microphotographs of neurons in $\boldsymbol{A}$ and $\boldsymbol{B}$. Scale bar, $20 \mu \mathrm{m}$. These neurons labeled with neurobiotin ( $\mathrm{Nb}$; red) are also immunoreactive for (hAT (green). $\boldsymbol{E}, \boldsymbol{F}$, Microphotographs at lower magnification showing that the neurons are located in areas with a high density of ChAT + neurons, i.e., in a dorsoventral band that expands laterally to the midline $(\boldsymbol{A})$ and in the ventral part of the vDB $(\boldsymbol{B})$. Scale bar, $50 \mu \mathrm{m}$.

Table 2. Patterns of discharge of neurons classified according to their neurochemical phenotype and the states of vigilance

\begin{tabular}{|c|c|c|c|c|}
\hline & & W & SWS & REMs \\
\hline GAD + & Burst-firing & $29.0 \pm 7.0$ & $37.7 \pm 10$ & $45.7 \pm 14$ \\
\hline \multirow[t]{5}{*}{$n=13$} & $n=3$ & $3 / 3$ & $3 / 3$ & $3 / 3$ \\
\hline & Cluster-firing & $16.5 \pm 4.8$ & $10.6 \pm 7.1$ & 9.0 \\
\hline & $n=6$ & $6 / 6$ & $6 / 6$ & $1 / 6$ \\
\hline & Tonic-firing & $23.3 \pm 2.9$ & $25.4 \pm 4.2$ & 33.0 \\
\hline & $n=4$ & $4 / 4$ & $4 / 4$ & $1 / 4$ \\
\hline$P V / G A D+$ & Burst-firing & $32.3 \pm 6.4$ & $40.5 \pm 8.1$ & $45.1 \pm 6.5$ \\
\hline \multirow[t]{3}{*}{$n=7$} & $n=6$ & $6 / 6$ & $6 / 6$ & $5 / 6$ \\
\hline & Cluster-firing & 19.0 & 24.0 & \\
\hline & $n=1$ & $1 / 1$ & $1 / 1$ & \\
\hline ChAT + & Slow-firing & $0.4 \pm 0.1$ & $1.9 \pm 1.3$ & \\
\hline$n=3$ & $n=3$ & $3 / 3$ & $3 / 3$ & \\
\hline Undetermined & Tonic-firing & $28.5 \pm 2.5$ & $31.5 \pm 8.5$ & \\
\hline \multirow[t]{3}{*}{$n=5$} & $n=2$ & $2 / 2$ & $2 / 2$ & \\
\hline & Slow-firing & $0.7 \pm 0.3$ & $2.2 \pm 1.3$ & 0.3 \\
\hline & $n=3$ & $3 / 3$ & $3 / 3$ & $1 / 3$ \\
\hline
\end{tabular}

Unanesthetized rats. Bolded values indicate the number of neurons observed during the corresponding vigilance state. Other values are average discharge rates (spikes/s) \pm SEM. Note that the burst-firing neurons are listed in the three columns, although they temporarily lost their rhythmicity during SWS.

(Borhegyi et al., 2004). The $18 \mathrm{GAD}+$ cells (15 from anesthetized and three from unanesthetized rats) were distributed in almost equivalent proportions between $\mathrm{T}$ type $(45.5 \%)$ and $\mathrm{P}$ type (54.5\%). The $18 \mathrm{PV} / \mathrm{GAD}+(12$ from anesthetized and six from unanesthetized rats) were more often of T type (77.7\%) than of $\mathrm{P}$ type $(22.2 \%)$. However, the distribution of $\mathrm{T}$ and $\mathrm{P}$ types in $\mathrm{GAD}+$ and PV/GAD + did not differ significantly $\left(p=0.28 ; \chi^{2}\right.$ test). In the unanesthetized condition, an individual neuron maintained the same phase preference during $\mathrm{W}$ and REMs. The correlation between phase preference and burst duration was also investigated. Burst length fell between 40 and $150 \mathrm{~ms}$. The cutoff between short and long burst was $75 \mathrm{~ms}$ (median of the distribution). The distribution of $\mathrm{T}$ and $\mathrm{P}$ types did not differ between short-burst neurons (11 T and $7 \mathrm{P}$ ) and long-burst neurons (12T and $7 \mathrm{P})$. With a more stringent distinction between short-burst ( $<55 \mathrm{~ms} ; 8$ cells) and long-burst ( $>100 \mathrm{~ms} ; 8$ cells), the repartition of $\mathrm{T}$ and $\mathrm{P}$ types was similar in both groups (6 T and $2 \mathrm{P}$ ). Last, types $\mathrm{T}$ and $\mathrm{P}$ bursting cells did not show preferential location in MS-DB.

\section{Discussion}

The present study documents, for the first time, the discharge properties of immunohistochemically identified MS-DB neurons in vivo, in unanesthetized rats. Our results indicate that the GABAergic neurons display tonic, cluster, or burst-firing patterns of discharge, depending on the vigilance states. The $\mathrm{RB}$ activity, observed at theta frequency during wakefulness and REMs, is more pronounced in GABAergic neurons that contain PV. Burst-firing neurons take on a tonic firing during SWS, and their discharge rates remain remarkably high across the sleep/wake cycle. None of the neurons with these functional properties have been found to be cholinergic. Instead, all of the identified cholinergic neurons have a low discharge rate, even in the presence of hippocampal theta. Undetermined neurons are not burstfiring but either tonic or slow-firing neurons. Results obtained in anesthetized rats also indicate that the theta-related RB activity is a property of GABAergic neurons and that the cholinergic neurons are slow firing. Undetermined neurons display various patterns of discharge, except the burst firing.

Although cholinergic neurons are numerous in MS-DB and easily revealed by ChAT immunochemistry (Kiss et al., 1990; Gritti et al., 1993), only a few neurobiotinlabeled neurons were found immunoreactive for ChAT ( 8 of 90). This rareness is probably attributable to the fact that the very low discharge rate of cholinergic cells limits their detection. In the light of the present results, one would assume that experiments focused on slow-firing cells would have allowed labeling of a larger number of cholinergic neurons, especially in the ventral part of the vertical limb of DB, which contains a higher proportion of cholinergic neurons (Amaral and Kurz, 1985; Kiss et al., 1990). The cholinergic neurons identified were few, but all had the same pattern of discharge, suggesting that our findings give reliable information on their firing properties.

Cluster-firing neurons have been described previously in the MS-DB of unanesthetized rats, but their neurochemical identity remains unknown (Rank, 1976; Sweeney et al., 1992). In the present study, we showed for the first time that a sizeable number of tonic and cluster-firing neurons was GAD + . They were observed both in anesthetized and unanesthetized rats where they displayed high firing rates during $\mathrm{W}$ but also during SWS and REMs. Few data are available on GABAergic MS-DB neurons that 
do not contain PV, but it seems that they are local neurons in their large majority (Kermer et al., 1995). It would be worth undertaking additional studies to elucidate the functions of these neurons, which retain high firing rates across the sleepwake cycle.

Glutamatergic neurons have been identified in MS-DB, some as local cells in position to exert an excitatory effect on surrounding GABAergic and cholinergic neurons (Hajszan et al., 2004; Colom et al., 2005; Manseau et al., 2005), others as septohippocampal neurons, which probably take a part in hippocampal theta generation (Sotty et al., 2003; Hajszan et al., 2004; Colom et al., 2005). An in vitro study showed that projection neurons that expressed the vesicular transporters of glutamate (VGLUT1 or VGLUT2) were slowfiring but that nearly half of them exhibited cluster firing in response to a prolonged depolarization (Sotty et al., 2003). Interestingly, our data show that 10 of 14 undetermined neurons $(71 \%)$ are slow firing/cluster firing, suggesting that they might be glutamatergic. Additional in vivo studies using juxtacellular labeling would be warranted to determine the discharge properties of identified glutamatergic MS-DB neurons.

PV neurons, recently identified in MS-DB of urethane anesthetized rats, display a theta-related bursting activity (Borhegyi et al., 2004; Bassant et al., 2005). They play a crucial role in theta generation via their rhythmic inputs to hippocampal GABAergic interneurons that, in turn, phasically disinhibit the pyramidal cells (Freund and Antal, 1988; Toth et al., 1997). The present study demonstrates that the PV/GAD + neurons discharge rhythmic bursts during wakefulness and REMs. They exhibit high discharge rates, a property that may be caused by the calcium-buffering properties of PV (Morris et al., 1999). We also show that many bursting neurons are GABAergic but do not express PV. It remains to understand how these neurons participate in the septal rhythmicity. Stewart and Fox (1989) reported on two populations of bursting MS-DB neurons both identified as projecting to the hippocampus. Those that retained burst firing after systemic injection of atropine had higher firing rates and more spikes per burst compared with those that lost their rhythmicity. The number of spikes per burst in each of these groups matches closely our own estimation for PV/GAD + and $\mathrm{GAD}+$ neurons. One might hypothesize that the two types of bursting neurons described by Stewart and Fox (1989) correspond to the $\mathrm{PV} / \mathrm{GAD}+$ and the GAD + neurons identified in our study.

A previous study on phase relationships of septal PV + bursting neurons to hippocampal theta showed an equivalent repartition of the cells into peak- or trough-preferring groups and a trend for long-burst neurons to be in phase with the peak of theta wave (Borhegyi et al., 2004). Concerning the first point, we found
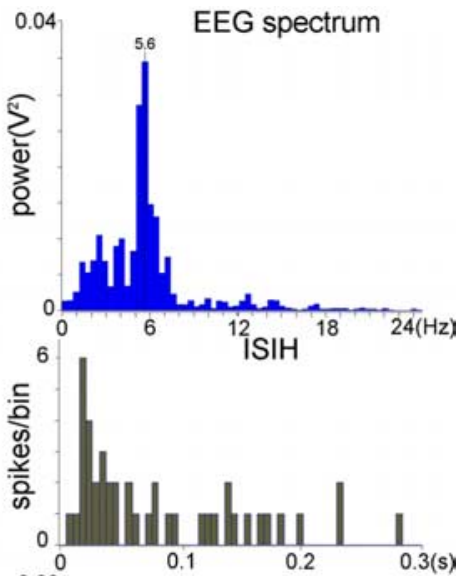

SWS
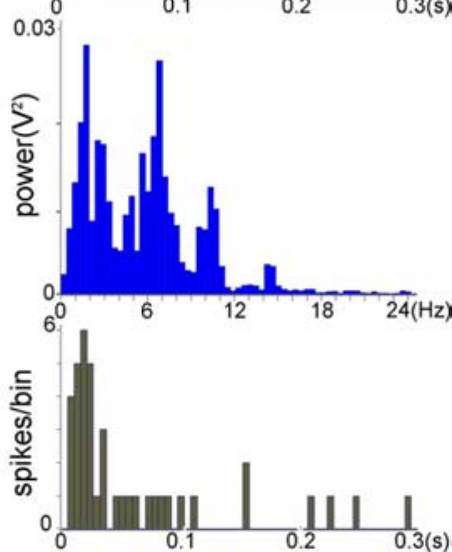

\section{$\mathrm{Nb}$}

GAD

$\mathrm{Nb} / \mathrm{GAD}$

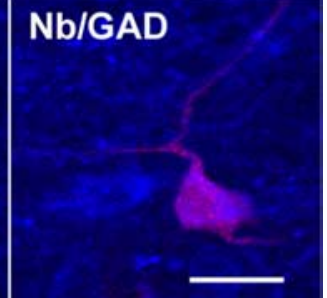

Figure 4. Discharge profile of a GAD + cluster-firing neuron recorded in an unanesthetized rat $(\boldsymbol{A})$. Note that the pattern of discharge is maintained when the vigilance shifts from W to SWS. $\boldsymbol{B}$, Single confocal sections showing the neuron $\boldsymbol{A}$ labeled with neurobiotin (Nb; red). This neuron was also immunoreactive for GAD (blue). Scale bar, $20 \mu \mathrm{m}$. The red dot on the frontal section of MS-DB shows the location of the neuron.

a similar result for the GAD + neurons, whereas $\mathrm{PV} / \mathrm{GAD}+$ neurons were more often related with the trough of theta. For the second point, the phase preference and the length of the burst did not appear to be linked. Such differences might be explained by methodological considerations (i.e., signal analysis or electrode location). They might also mean that the phase relationships of MS-DB bursting neurons to hippocampal theta are complex as attested by the frequent observation that two neurons recorded during the same electrode penetration might display similar bursting characteristics but different theta phase preferences.

Stewart and Fox (1990) proposed that the atropine-resistant bursting neurons were GABAergic, whereas the atropinesensitive were cholinergic. According to their model, both cholinergic and GABAergic cells fire in rhythmic bursts that entrain hippocampal interneurons. This hypothesis was mostly accepted (Smythe et al., 1992; Lee et al., 1994; Brazhnik and Fox, 1997; King et al., 1998). Having observed a loss of bursting activity in MS-DB after selective lesion of cholinergic septohippocampal neurons, we also concluded that the cholinergic neurons contributed to the rhythmically bursting pool of MS-DB neurons (Apar- 


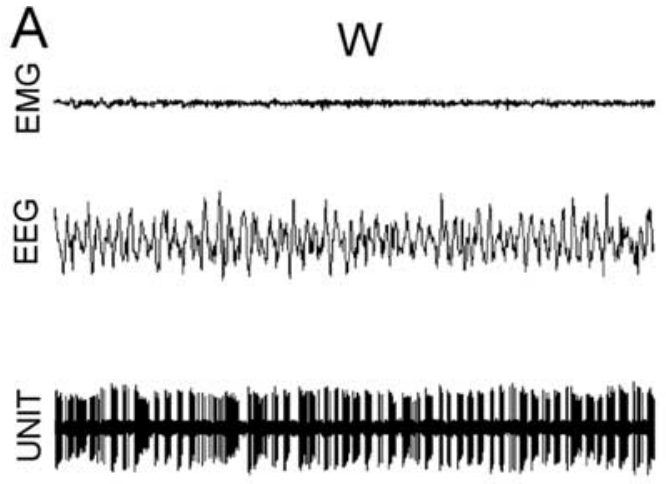

SWS

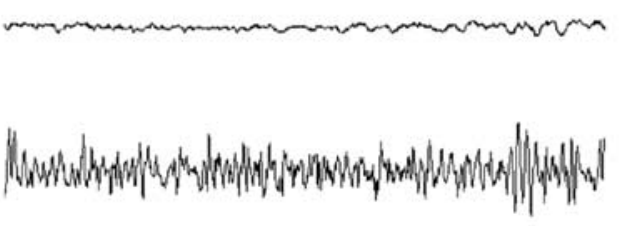

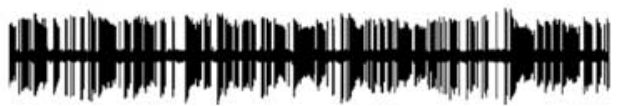

REMs

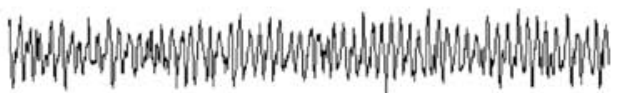

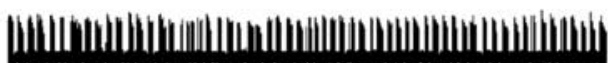

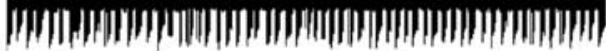

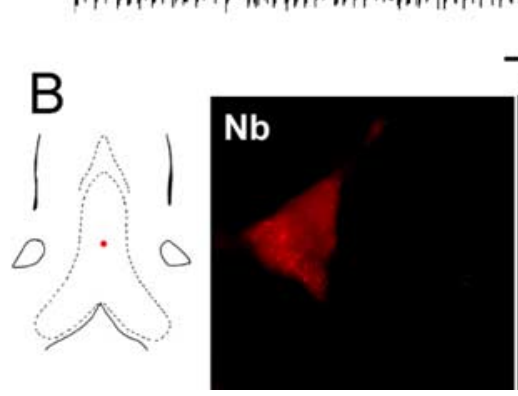
$0.5 \mathrm{mV}$
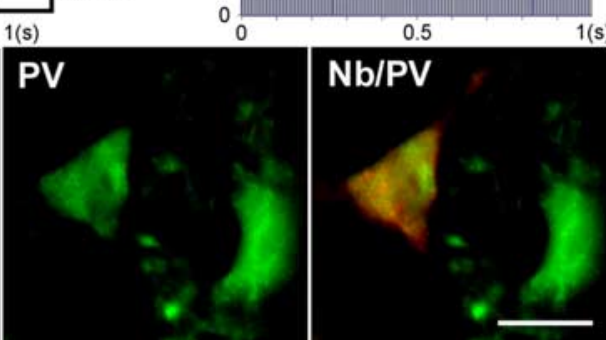

Figure 5. Discharge profile of a PV/GAD + bursting neuron recorded across the sleep-wake cycle $(\boldsymbol{A})$. Note that the rhythmically bursting activity present during $W$ is virtually abolished during SWS but is accentuated during REMs, as shown by the oscillatory profile of HAC and the high value of RI. $\boldsymbol{B}$, The neuron labeled with neurobiotin (red) is also immunoreactive for PV (green). Scale bar, $20 \mu \mathrm{m}$. Note that the neuron is located in the midline of MS (red dot), an area especially rich in PV neurons.
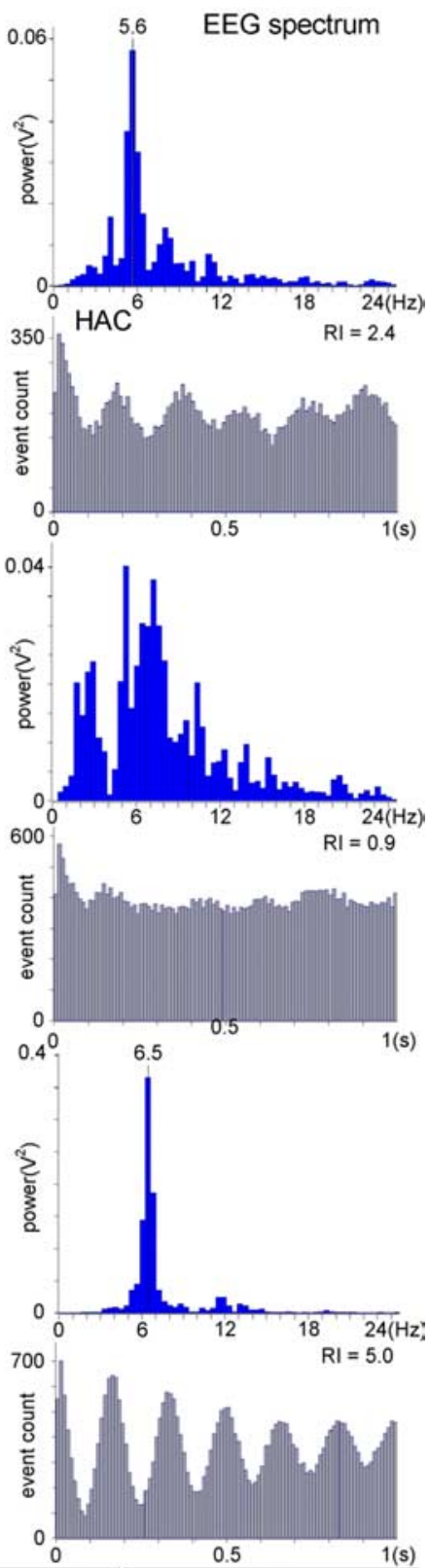

tis et al., 1998). The present results call this hypothesis into question. They strongly suggest that the MS-DB cholinergic neurons have a low discharge rate and do not display theta-related burst firing or tonic activity even during wakefulness. None of the four cholinergic neurons identified in unanesthetized rats could be recorded during REMs. However, it is unlikely that these slowfiring neurons recorded during wakefulness would have turned to burst firing or high tonic firing during REMs. We have shown previously, indeed, that induction of bursting activity by external stimuli or arousal changes was possible only in fast-spiking neurons (Sweeney et al., 1992). Our in vivo results are consistent with in vitro data, which show that MS-DB cholinergic neurons have intrinsic properties that limit the likelihood of high-frequency firing and bursting activity (Markram and Segal, 1990; Gorelova and Reiner, 1996; Sotty et al., 2003).

The properties of MS-DB cholinergic neurons observed in the present study are in marked contrast with those of cholinergic neurons recorded in the nucleus basalis magnocellularis (NBM) of unanesthetized rats. NBM cholinergic neurons discharged in bursts at maximal rates during waking and REMs and could therefore contribute directly to the generation of cortical theta activity (Lee et al., 2005). Once more, the in vivo data are consistent with the in vitro ones, which show that NBM cholinergic neurons have intrinsic membrane properties that allow for fast activity on rhythmic or tonic modes (Khateb et al., 1992). Together, these data show that cholinergic neurons, although located in adjacent regions of the basal forebrain, exhibit different firing properties, suggesting differences in the role of ACh in the cortex and hippocampus.

Extensive data show that ACh has an excitatory effect on GABAergic and glutamatergic neurons within MS-DB (Wu et al., 2000, 2003; Manseau et al., 2005) and that it plays a key role in hippocampal theta generation through excitation of both principal cells and interneurons (Buzsaki, 2002). Also, considerable evidence shows the implication of the septohippocampal ACh in learning and memory functions, at least as a modulator factor (for review, see Parent and Baxter, 2006). The finding that MS-DB cholinergic neurons have a low firing rate is unexpected and questions how ACh is released to ensure these various functions. Ultrastructural considerations provide a possible, although limited, explanation. The density of cholinergic innervation is very high in the hippocampus, but it is predominantly asynaptic in nature (Umbriaco et al., 1995). It is assumed that such innervation exerts its effects by diffuse transmission, in addition to synaptic transmission (Descarries et al., 2004). Diffuse transmission would maintain a low ambient level of ACh that would reinforce the efficiency of depolarizing potentials so that cholinergic neurons could act on their targets even in the case of low-frequency discharge.

In conclusion, our in vivo study establishes undoubtedly that the bursting activity in MS-DB is characteristic of the GABAergic neurons, expressing or not PV. The original observation that 
cholinergic neurons have a low firing rate raises the intriguing question of the mechanisms of action of ACh in the septohippocampal system.

\section{References}

Amaral DG, Kurz J (1985) An analysis of the origins of the cholinergic and noncholinergic septal projections to the hippocampal formation of the rat. J Comp Neurol 240:37-59.

Apartis E, Poindessous-Jazat FR, Lamour YA, Bassant MH (1998) Loss of rhythmically bursting neurons in rat medial septum following selective lesion of septohippocampal cholinergic system. J Neurophysiol 79:1633-1642.

Bassant MH, Simon A, Poindessous-Jazat F, Csaba Z, Epelbaum J, Dournaud P (2005) Medial septal GABAergic neurons express the somatostatin sst2A receptor: functional consequences on unit firing and hippocampal theta. J Neurosci 25:2032-2041.

Borhegyi Z, Varga V, Szilagyi N, Fabo D, Freund TF (2004) Phase segregation of medial septal GABAergic neurons during hippocampal theta activity. J Neurosci 24:8470-8479.

Brazhnik ES, Fox SE (1997) Intracellular recordings from medial septal neurons during hippocampal theta rhythm. Exp Brain Res 114:442-453.

Brazhnik ES, Fox SE (1999) Action potentials and relations to the theta rhythm of medial septal neurons in vivo. Exp Brain Res 127:244-258.

Buzsaki G (2002) Theta oscillations in the hippocampus. Neuron 33:325-340.

Colom LV, Castaneda MT, Reyna T, Hernandez S, Garrido-Sanabria E (2005) Characterization of medial septal glutamatergic neurons and their projection to the hippocampus. Synapse 58:151-164.

Descarries L, Mechawar N, Aznavour N, Watkins KC (2004) Structural determinants of the roles of acetylcholine in the cerebral cortex. Prog Brain Res 145:45-58.

Dutar P, Lamour Y, Rascol O, Jobert A (1986) Septo-hippocampal neurons in the rat: further study of their physiological and pharmacological properties. Brain Res 365:325-334.

Freund TF (1989) GABAergic septohippocampal neurons contain parvalbumin. Brain Res 478:375-381.

Freund TF, Antal M (1988) GABA-containing neurons in the septum control inhibitory interneurons in the hippocampus. Nature 336:170-173.

Frotscher M, Leranth C (1985) Cholinergic innervation of the rat hippocampus as revealed by choline acetyltransferase immunocytochemistry: a combined light and electron microscopic study. J Comp Neurol 239:237-246.

Gorelova N, Reiner PB (1996) Role of the afterhyperpolarization in control of discharge properties of septal cholinergic neurons in vitro. J Neurophysiol 75:695-706.

Griffith WH, Matthews RT (1986) Electrophysiology of AChE-positive neurons in basal forebrain slices. Neurosci Lett 71:169-174.

Gritti I, Mainville L, Jones BE (1993) Codistribution of GABA with acetylcholine-synthesizing neurons in the basal forebrain of the rat. J Comp Neurol 329:438-457.

Hajszan T, Alreja M, Leranth C (2004) Intrinsic vesicular glutamate transporter 2-immunoreactive input to septohippocampal parvalbumincontaining neurons: novel glutamatergic local circuit cells. Hippocampus 14:499-509.

Kermer P, Naumann T, Bender R, Frotscher M (1995) Fate of GABAergic septohippocampal neurons after fimbria-fornix transection as revealed by in situ hybridization for glutamate decarboxylase mRNA and parvalbumin immunohistochemistry. J Comp Neurol 362:385-399.

Khateb A, Muhlethaler M, Alonso A, Serafin M, Mainville L, Jones BE (1992) Cholinergic nucleus basalis neurons display the capacity for rhythmic bursting activity mediated by low-threshold calcium spike. Neuroscience 51:489-494.

King C, Recce M, O'Keefe J (1998) The rhythmicity of cells of the medial septum/diagonal band of Broca in the awake freely moving rat: relationships with behaviour and hippocampal theta. Eur J Neurosci 10:464-477.

Kiss J, Patel AJ, Baimbridge KG, Freund TF (1990) Topographical localization of neurons containing parvalbumin and choline acetyltransferase in the medial septum-diagonal band region of the rat. Neuroscience 36:61-72.

Köhler C, Chan-Palay V, Wu JY (1984) Septal neurons containing glutamic acid decarboxylase immunoreactivity project to the hippocampal region in the rat brain. Anat Embryol (Berl) 169:41-44.

Lee MG, Chrobak JJ, Sik A, Wiley RG, Buzsaki G (1994) Hippocampal theta activity following selective lesion of the septal cholinergic system. Neuroscience 62:1033-1047.

Lee MG, Hassani OK, Alonso A, Jones BE (2005) Cholinergic basal forebrain neurons burst with theta during waking and paradoxical sleep. J Neurosci 25:4365-4369.

Manns ID, Mainville L, Jones BE (2001) Evidence for glutamate, in addition to acetylcholine and GABA, neurotransmitter synthesis in basal forebrain neurons projecting to the entorhinal cortex. Neuroscience 107:247-263.

Manseau F, Danik M, Williams S (2005) A functional glutamatergic neurone network in the medial septum and diagonal band area. J Physiol (Lond) 566:865-884.

Markram H, Segal M (1990) Electrophysiological characteristics of cholinergic and non-cholinergic neurons in the rat medial septum-diagonal band complex. Brain Res 513:171-174.

Matthews RT, Lee WL (1991) A comparison of extracellular and intracellular recordings from medial septum/diagonal band neurons in vitro. Neuroscience 42:451-462.

Morris NP, Harris SJ, Henderson Z (1999) Parvalbumin-immunoreactive, fast-spiking neurons in the medial septum/diagonal band complex of the rat: intracellular recordings in vitro. Neuroscience 92:589-600.

Parent MB, Baxter M (2006) Septohippocampal acetylcholine: involved in but not necessary for learning and memory? Learn Mem 11:9-20.

Paxinos G, Watson C (1986) The rat brain in stereotaxic coordinates atlas. Sydney: Academic.

Perkel DH, Gerstein GL, Moore GP (1967) Neuronal spike trains and stochastic point processes. I. The single spike train. Biophys J 7:391-418.

Pinault D (1996) A novel single-cell staining procedure performed in vivo under electrophysiological control: morpho-functional features of juxtacellularly labeled thalamic cells and other central neurons with biocytin or neurobiotin. J Neurosci Methods 65:113-136.

Rank J (1976) Behavioral correlates and firing repertoires of neurons in septal nuclei in unrestrained rats. In: The septal nuclei (DeFrance JF, ed), pp 423-462. New York: Plenum.

Smythe JW, Colom LV, Bland BH (1992) The extrinsic modulation of hippocampal theta depends on the coactivation of cholinergic and GABAergic medial septal inputs. Neurosci Biobehav Rev 16:289-308.

Sotty F, Danik M, Manseau F, Laplante F, Quirion R, Williams S (2003) Distinct electrophysiological properties of glutamatergic, cholinergic and GABAergic rat septohippocampal neurons: novel implications for hippocampal rhythmicity. J Physiol (Lond) 551:927-943.

Souliere F, Urbain N, Gervasoni D, Schmitt P, Guillemort C, Fort P, Renaud B, Luppi PH, Chouvet G (2000) Single-unit and polygraphic recordings associated with systemic or local pharmacology: a multi-purpose stereotaxic approach for the awake, anaesthetic-free, and head-restrained rat. J Neurosci Res 61:88-100.

Stewart M, Fox SE (1989) Two populations of rhythmically bursting neurons in rat medial septum are revealed by atropine. J Neurophysiol 61:982-993.

Stewart M, Fox SE (1990) Do septal neurons pace the hippocampal theta rhythm? Trends Neurosci 13:163-168.

Sweeney JE, Lamour Y, Bassant MH (1992) Arousal-dependent properties of medial septal neurons in the unanesthetized rat. Neuroscience 48:353-362.

Toth K, Freund TF, Miles R (1997) Disinhibition of rat hippocampal pyramidal cells by GABAergic afferents from the septum. J Physiol (Lond) 500:463-474.

Umbriaco D, Garcia S, Beaulieu C, Descarries L (1995) Relational features of acetylcholine, noradrenaline, serotonin and GABA axon terminals in the stratum radiatum of adult rat hippocampus (CA1). Hippocampus 5:605-620.

Wu M, Shanabrough M, Leranth C, Alreja M (2000) Cholinergic excitation of septohippocampal GABA but not cholinergic neurons: implications for learning and memory. J Neurosci 20:3900-3908.

Wu M, Hajszan T, Leranth C, Alreja M (2003) Nicotine recruits a local glutamatergic circuit to excite septohippocampal GABAergic neurons. Eur J Neurosci 18:1155-1168. 